Acta Crystallographica Section E

Structure Reports

Online

ISSN 1600-5368

\section{Penikisite, $\mathrm{BaMg}_{2} \mathrm{Al}_{2}\left(\mathrm{PO}_{4}\right)_{3}(\mathrm{OH})_{3}$, isostructural with bjarebyite}

\section{Michael G. Bowman,* Robert T. Downs and Hexiong Yang}

Department of Geosciences, University of Arizona, 1040 E. 4th Street, Tucson, Arizona 85721-0077, USA

Correspondence e-mail: bowman90@email.arizona.edu

Received 6 December 2012; accepted 23 December 2012

Key indicators: single-crystal X-ray study; $T=293 \mathrm{~K}$; mean $\sigma(\mathrm{P}-\mathrm{O})=0.002 \AA$. disorder in main residue; $R$ factor $=0.015 ; w R$ factor $=0.039$; data-to-parameter ratio $=16.6$

The bjarebyite group of minerals, characterized by the general formula $\mathrm{BaX}_{2} Y_{2}\left(\mathrm{PO}_{4}\right)_{3}(\mathrm{OH})_{3}$, with $X=\mathrm{Mg}, \mathrm{Fe}^{2+}$ or $\mathrm{Mn}^{2+}$, and $Y=\mathrm{Al}$ or $\mathrm{Fe}^{3+}$, includes five members: bjarebyite $\mathrm{BaMn}_{2+}{ }_{2} \mathrm{Al}_{2^{-}}$ $\left(\mathrm{PO}_{4}\right)_{3}(\mathrm{OH})_{3}$, johntomaite $\mathrm{BaFe}_{2}^{2+}{ }_{2} \mathrm{Fe}^{3+}{ }_{2}\left(\mathrm{PO}_{4}\right)_{3}(\mathrm{OH})_{3}$, kulanite $\mathrm{BaFe}_{2}^{2+}{ }_{2} \mathrm{Al}_{2}\left(\mathrm{PO}_{4}\right)_{3}(\mathrm{OH})_{3}$, penikisite $\mathrm{BaMg}_{2} \mathrm{Al}_{2}\left(\mathrm{PO}_{4}\right)_{3^{-}}$ $(\mathrm{OH})_{3}$, and perloffite $\mathrm{BaMn}^{2+}{ }_{2} \mathrm{Fe}^{3+}{ }_{2}\left(\mathrm{PO}_{4}\right)_{3}(\mathrm{OH})_{3}$. Thus far, the crystal structures of all minerals in the group, but penikisite, have been determined. The present study reports the first structure determination of penikisite (barium dimagnesium dialuminium triphosphate trihydroxide) using single-crystal X-ray diffraction data of a crystal from the type locality, Mayo Mining District, Yukon Territory, Canada. Penikisite is isotypic with other members of the bjarebyite group with space group $P 2_{1} / m$, rather than triclinic ( $P 1$ or $\left.P \overline{1}\right)$, as previously suggested. Its structure consists of edge-shared $\left[\mathrm{AlO}_{3}(\mathrm{OH})_{3}\right]$ octahedral dimers linking via corners to form chains along [010]. These chains are decorated with $\mathrm{PO}_{4}$ tetrahedra (one of which has site symmetry $m$ ) and connected along [100] via edge-shared $\left[\mathrm{MgO}_{5}(\mathrm{OH})\right]$ octahedral dimers and eleven-coordinated $\mathrm{Ba}^{2+}$ ions (site symmetry $m$ ), forming a complex three-dimensional network. $\mathrm{O}-\mathrm{H} \cdots \mathrm{O}$ hydrogen bonding provides additional linkage between chains. Microprobe analysis of the crystal used for data collection indicated that $\mathrm{Mn}$ substitutes for $\mathrm{Mg}$ at the $1.5 \%$ (apfu) level.

\section{Related literature}

For penikisite, see: Mandarino et al. (1977). For other mineral members in the bjarebyite group, see: Moore \& Araki (1974); Cooper \& Hawthorne (1994); Kolitsch et al. (2000); Elliott \& Willis (2011). For the definition of polyhedral distortion, see: Robinson et al. (1971).

\section{Experimental}

Crystal data

$\mathrm{BaMg}_{2} \mathrm{Al}_{2}\left(\mathrm{PO}_{4}\right)_{3}(\mathrm{OH})_{3}$

$M_{r}=576.77$

Monoclinic, $P 2_{1} / m$

$a=8.9577$ (4) А

$b=12.0150(5) \AA$

$c=4.9079(2) \AA$

$\beta=100.505(2)^{\circ}$

Data collection

Bruker APEXII CCD

diffractometer

Absorption correction: multi-scan (SADABS; Sheldrick, 2005)

$T_{\min }=0.676, T_{\max }=0.704$

Refinement

$R\left[F^{2}>2 \sigma\left(F^{2}\right)\right]=0.015$

$w R\left(F^{2}\right)=0.039$

$S=1.14$

1970 reflections

119 parameters

Table 1

Selected bond lengths (Å).

\begin{tabular}{llll}
\hline $\mathrm{Mg}-\mathrm{O} 7^{\mathrm{i}}$ & $2.0591(11)$ & $\mathrm{Al}-\mathrm{O} H 8$ & $1.9477(7)$ \\
$\mathrm{Mg}-\mathrm{O} 1^{\text {ii }}$ & $2.0864(10)$ & $\mathrm{P} 1-\mathrm{O} 2$ & $1.5232(14)$ \\
$\mathrm{Mg}-\mathrm{O} 2^{\text {iii }}$ & $2.1227(10)$ & $\mathrm{P} 1-\mathrm{O} 1$ & $1.5278(15)$ \\
$\mathrm{Mg}-\mathrm{OH} 9^{\text {iv }}$ & $2.1729(11)$ & $\mathrm{P} 1-\mathrm{O} 3$ & $1.5321(10)$ \\
$\mathrm{Mg}-\mathrm{O}^{\mathrm{v}}$ & $2.2090(12)$ & $\mathrm{P} 1-\mathrm{O} 3^{\text {ix }}$ & $1.5321(10)$ \\
$\mathrm{Al}-\mathrm{O} 3^{\text {vi }}$ & $1.8523(11)$ & $\mathrm{P} 2-\mathrm{O} 4$ & $1.5083(11)$ \\
$\mathrm{Al}-\mathrm{O} 6$ & $1.9080(11)$ & $\mathrm{P} 2-\mathrm{O} 7$ & $1.5272(11)$ \\
$\mathrm{Al}-\mathrm{O} 5^{\text {vii }}$ & $1.9287(10)$ & $\mathrm{P} 2-\mathrm{O} 6$ & $1.5443(11)$ \\
$\mathrm{Al}-\mathrm{OH} 9$ & $1.9397(11)$ & $\mathrm{P} 2-\mathrm{O} 5$ & $1.5680(10)$ \\
$\mathrm{Al}-\mathrm{O} H 9^{\text {viii }}$ & $1.9440(11)$ & &
\end{tabular}

$\mathrm{Al}-\mathrm{OH} 9^{\mathrm{vii}}$

$1.9440(11)$

1 restraint

All H-atom parameters refined

$\Delta \rho_{\max }=0.72{\mathrm{e} \AA^{-3}}^{-3}$

$\Delta \rho_{\min }=-0.80 \mathrm{e}^{-3}$

Symmetry codes: (i) $-x+1, y-\frac{1}{2},-z+1$; (ii) $x, y-1, z-1$; (iii) $x, y-1, z$; (iv) $x,-y+\frac{1}{2}, z$; (v) $x,-y+\frac{1}{2}, z-1$; (vi) $-x,-y+1,-z+1$; (vii) $x, y, z-1$; (viii) $-x,-y+1,-z$; (ix) $x,-y+\frac{3}{2}, z$.

Table 2

Hydrogen-bond geometry $\left(\AA,{ }^{\circ}\right)$.

\begin{tabular}{lllll}
\hline$D-\mathrm{H} \cdots A$ & $D-\mathrm{H}$ & $\mathrm{H} \cdots A$ & $D \cdots A$ & $D-\mathrm{H} \cdots A$ \\
\hline $\mathrm{OH} 8-\mathrm{H} 1 \cdots \mathrm{O}^{\text {vii }}$ & $0.79(4)$ & $2.66(3)$ & $3.3180(16)$ & $142(1)$ \\
$\mathrm{OH} 9-\mathrm{H} 2 \cdots \mathrm{O} 3$ & $0.78(3)$ & $1.89(3)$ & $2.6512(13)$ & $166(3)$ \\
\hline
\end{tabular}

Symmetry code: (vii) $x, y, z-1$.

Data collection: APEX2 (Bruker, 2004); cell refinement: SAINT (Bruker, 2004); data reduction: $S A I N T$; $\operatorname{program}(\mathrm{s})$ used to solve structure: SHELXS97 (Sheldrick, 2008); program(s) used to refine structure: SHELXL97 (Sheldrick, 2008); molecular graphics: XtalDraw (Downs \& Hall-Wallace, 2003); software used to prepare material for publication: publCIF (Westrip, 2010).

The authors gratefully acknowledge support of this study by the Science Foundation Arizona.

Supplementary data and figures for this paper are available from the IUCr electronic archives (Reference: HB7009). 


\section{inorganic compounds}

\section{References}

Bruker (2004). APEX2 and SAINT. Bruker AXS Inc., Madison, Wisconsin, USA.

Cooper, M. \& Hawthorne, F. C. (1994). Can. Mineral. 32, 15-19.

Downs, R. T. \& Hall-Wallace, M. (2003). Am. Mineral. 88, 247-250.

Elliott, P. \& Willis, A. C. (2011). Mineral. Mag. 75, 317-325.

Kolitsch, U., Pring, A. \& Tiekink, E. R. T. (2000). Mineral. Petrol. 70, 1-14.
Mandarino, J. A., Sturman, B. D. \& Corlett, M. I. (1977). Can. Mineral. 15, $393-$ 395.

Moore, P. B. \& Araki, T. (1974). Am. Mineral. 59, 567-572.

Robinson, K., Gibbs, G. V. \& Ribbe, P. H. (1971). Science, 172, 567-570.

Sheldrick, G. M. (2005). SADABS. University of Göttingen, Germany.

Sheldrick, G. M. (2008). Acta Cryst. A64, 112-122.

Westrip, S. P. (2010). J. Appl. Cryst. 43, 920-925. 


\section{supporting information}

Acta Cryst. (2013). E69, i4-i5 [doi:10.1107/S1600536812051793]

\section{Penikisite, $\mathrm{BaMg}_{2} \mathrm{Al}_{2}\left(\mathrm{PO}_{4}\right)_{3}(\mathrm{OH})_{3}$, isostructural with bjarebyite}

\section{Michael G. Bowman, Robert T. Downs and Hexiong Yang}

\section{S1. Comment}

The bjarebyite group of minerals is characterized by the general chemical formula $\mathrm{Ba} X_{2} Y_{2}\left(\mathrm{PO}_{4}\right)_{3}(\mathrm{OH})_{3}$, where $X=\mathrm{Mn}^{2+}$, $\mathrm{Fe}^{2+}$ or $\mathrm{Mg}$ and $Y=\mathrm{Al}$ or $\mathrm{Fe}^{3+}$, and includes five members: bjarebyite $\mathrm{BaMn}^{2+}{ }_{2} \mathrm{Al}_{2}\left(\mathrm{PO}_{4}\right)_{3}(\mathrm{OH})_{3}$, johntomaite $\mathrm{BaFe}^{2+}{ }_{2} \mathrm{Fe}^{3+}{ }_{2}\left(\mathrm{PO}_{4}\right)_{3}(\mathrm{OH})_{3}$, kulanite $\mathrm{BaFe}^{2+}{ }_{2} \mathrm{Al}_{2}\left(\mathrm{PO}_{4}\right)_{3}(\mathrm{OH})_{3}$, penikisite $\mathrm{BaMg}_{2} \mathrm{Al}_{2}\left(\mathrm{PO}_{4}\right)_{3}(\mathrm{OH})_{3}$, and perloffite $\mathrm{BaMn}_{2}^{2+}{ }_{2} \mathrm{Fe}^{3+}{ }_{2}\left(\mathrm{PO}_{4}\right)_{3}(\mathrm{OH})_{3}$. Except for penikisite, the crystal structures of all other minerals in the group have been determined (Moore and Araki, 1974; Cooper and Hawthorne, 1994; Kolitsch et al., 2000; Elliot \& Willis, 2011), which all possess space group $P 2_{1} / \mathrm{m}$. Penikisite was first described by Mandarino et al. (1977) as triclinic with space group $P 1$ or $P \overline{1}$ (albeit strongly pseudomonoclinic), based on the observation of asymmetric optical dispersion. Since then, no detailed crystallographic study on penikisite has been reported. In our efforts to understand the hydrogen bonding environments in minerals, we conducted a structure determination of penikisite from the type locality by means of single-crystal X-ray diffraction.

Penikisite is isotypic with other members of the bjarebyite group, with space group $P 2_{1} / \mathrm{m}$. Its structure consists of edgeshared $\left[\mathrm{AlO}_{3}(\mathrm{OH})_{3}\right]$ octahedral dimers connected via corners to form chains along [010]. These chains are decorated with $\mathrm{PO}_{4}$ tetrahedra and linked along [100] via edge-shared $\mathrm{MgO}_{5}(\mathrm{OH})$ octahedral dimers and eleven-coordinated $\mathrm{Ba}$ atoms to form a complex three-dimensional network (Figs. 1 and 2). The hydrogen bonding provides additional linkage between chains.

Similar to other minerals in the bjarebyite group, the $Y_{5}(\mathrm{OH})$ octahedra in penikisite are noticeably distorted, as measured by the octahedral angle variance (OAV) and quadratic elongation (OQE) (Robinson et al., 1971), which are 188 and 1.057, respectively. In contrast, the $\mathrm{OAV}$ and $\mathrm{OQE}$ values are 32 and 1.010 for the $\mathrm{XO}_{3}(\mathrm{OH})_{3}$ octahedra in penikisite. From penikisite to the Fe-analogue kulanite (Cooper and Hawthorne, 1994), and to the Mn-analogue bjarebyite (Moore and Araki, 1974), the average $X$-O distance increases from 2.117 to 2.146 , and to $2.162 \AA$, respectively, in accordance with the increase in the ionic radius in this site.

There are two hydrogen bonds in penikisite, one between OH8 and O6 [3.318 (2) $\AA]$ and the other between OH9 and O3 [2.651 (1) $\AA$ ], agreeing well with the results obtained by Elliott \& Willis (2011) from perloffite. However, Cooper and Hawthorne (1994) proposed a disorder model for H1 in kulanite. The H atoms were not located in the structure of bjarebyite (Moore and Araki, 1974) or johntomaite (Kolitsch et al., 2000).

\section{S2. Experimental}

The penikisite crystal used in this study is from the type locality, 16 miles north of the Hess River, Mayo Mining District, Yukon Territory, Canada and is in the collection of the RRUFF project (http://rruff.info/R060160), donated by Mark Mauthner. Its chemistry was determined with a CAMECA SX50 electron microprobe ( 8 analysis points), yielding the empirical chemical formula, calculated on the basis of $13.5 \mathrm{O}$ atoms, $\mathrm{Ba}_{1.00}\left(\mathrm{Mg}_{1.97} \mathrm{Mn}_{0.03}\right)_{\Sigma=2} \mathrm{Al}_{2.00}\left(\mathrm{P}_{1.00} \mathrm{O}_{4}\right)_{3}(\mathrm{OH})_{3}(\mathrm{OH}$ was estimated by charge balance and difference). 


\section{S3. Refinement}

The $\mathrm{H}$ atoms were located from difference Fourier syntheses and their positions refined freely with a fixed isotropic displacement $\left(U_{i s o}=0.03\right)$. The highest residual peak in the difference Fourier maps was located at $(0.4023,0.2932$, 0.2033), $0.71 \AA$ from $\mathrm{Ba}$, and the deepest hole at $(0.5192,1 / 4,0.3234), 0.63 \AA$ from Ba.

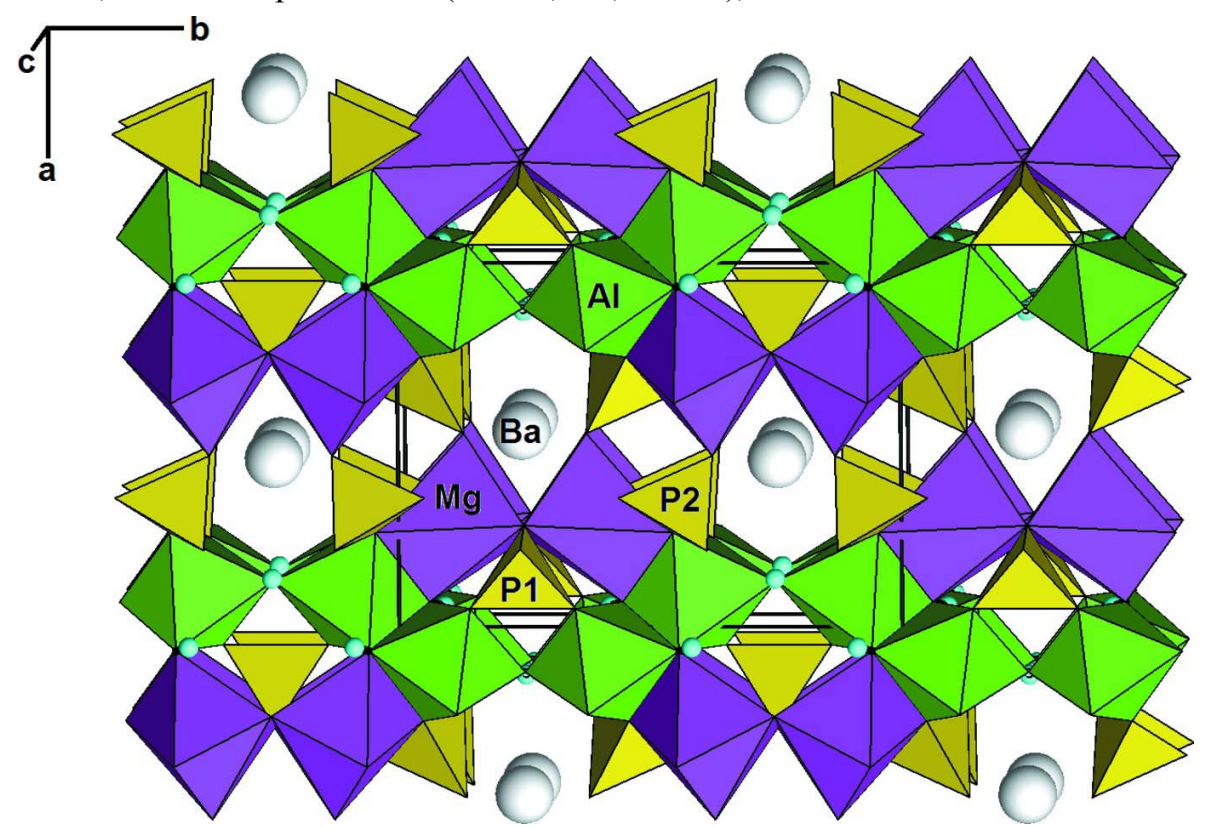

\section{Figure 1}

Crystal structure of penikisite in polyhedral representation. Large and small spheres represent $\mathrm{Ba}$ and $\mathrm{H}$ atoms, respectively. 


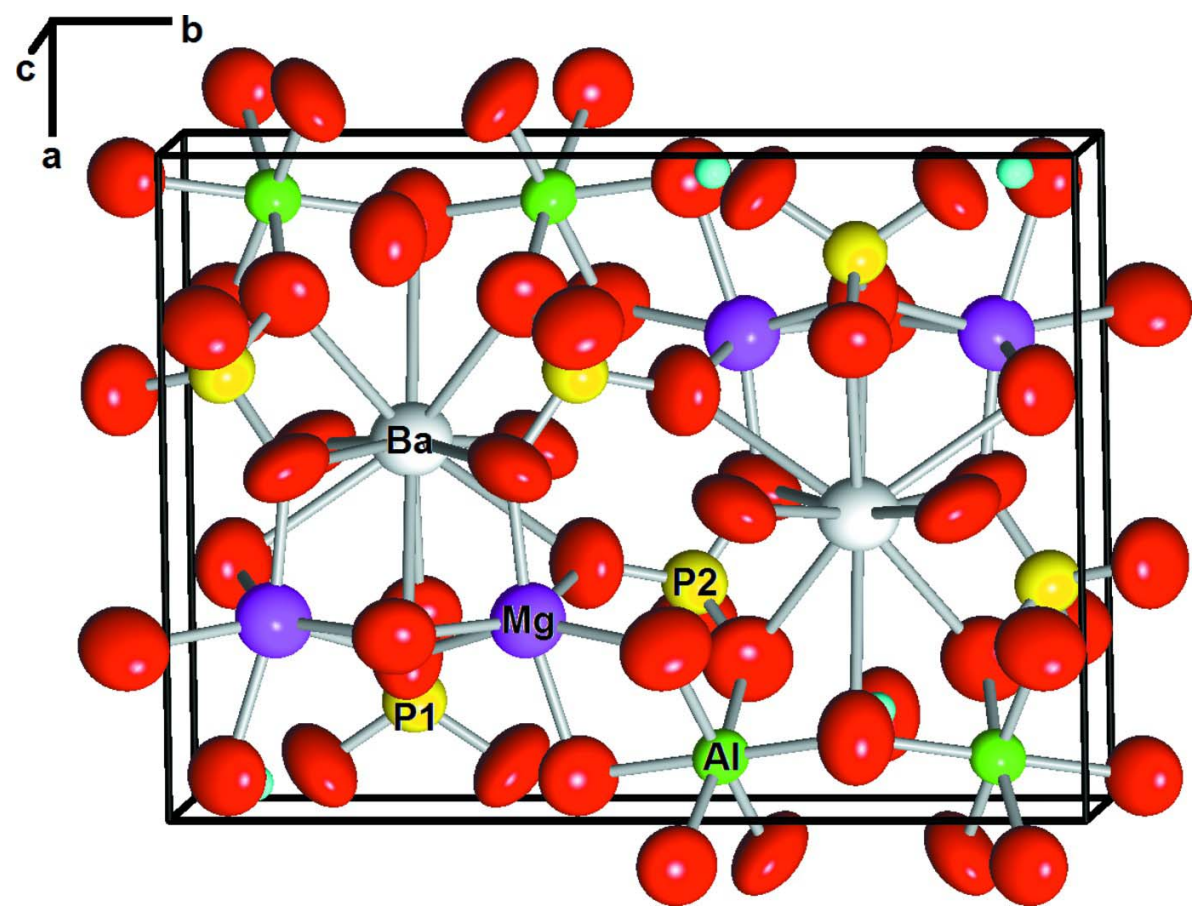

Figure 2

The crystal structure of penikisite, showing atoms, except for $\mathrm{H}$, with displacement ellipsoids at the $99 \%$ probability level. Gray, pink, green, yellow, and red ellipsoids represent $\mathrm{Ba}, \mathrm{Mg}, \mathrm{Al}, \mathrm{P}$, and $\mathrm{O}$ atoms, respectively. $\mathrm{H}$ atoms are given as turquoise spheres with an arbitrary radius.

\section{Barium dimagnesium dialuminium triphosphate trihydroxide}

\section{Crystal data}

$\mathrm{Al}_{4} \mathrm{H}_{6} \mathrm{Mg}_{3.94} \mathrm{Mn}_{0.06} \mathrm{O}_{30} \mathrm{P}_{6} \cdot 2(\mathrm{Ba})$

$M_{r}=576.77$

Monoclinic, $P 12_{1} / m 1$

Hall symbol: -P $2 \mathrm{yb}$

$a=8.9577$ (4) $\AA$

$b=12.0150(5) \AA$

$c=4.9079(2) \AA$

$\beta=100.505(2)^{\circ}$

$V=519.37(4) \AA^{3}$

$Z=2$

Data collection

Bruker APEXII CCD

diffractometer

Radiation source: fine-focus sealed tube

Graphite monochromator

$\varphi$ and $\omega$ scan

Absorption correction: multi-scan

(SADABS; Sheldrick 2005)

$T_{\min }=0.676, T_{\max }=0.704$
$F(000)=549$

nearly cube

$D_{\mathrm{x}}=3.688 \mathrm{Mg} \mathrm{m}^{-3}$

Mo $K \alpha$ radiation, $\lambda=0.71073 \AA$

Cell parameters from 6030 reflections

$\theta=2.9-32.6^{\circ}$

$\mu=4.72 \mathrm{~mm}^{-1}$

$T=293 \mathrm{~K}$

Cube, green

$0.09 \times 0.09 \times 0.08 \mathrm{~mm}$

7681 measured reflections

1970 independent reflections

1925 reflections with $I>2 \sigma(I)$

$R_{\text {int }}=0.020$

$\theta_{\text {max }}=32.6^{\circ}, \theta_{\min }=2.9^{\circ}$

$h=-13 \rightarrow 12$

$k=-15 \rightarrow 18$

$l=-7 \rightarrow 7$ 


\section{Refinement}

Refinement on $F^{2}$

Least-squares matrix: full

$R\left[F^{2}>2 \sigma\left(F^{2}\right)\right]=0.015$

$w R\left(F^{2}\right)=0.039$

$S=1.14$

1970 reflections

119 parameters

1 restraint

Primary atom site location: structure-invariant direct methods
Secondary atom site location: difference Fourier map

Hydrogen site location: difference Fourier map

All $\mathrm{H}$-atom parameters refined

$w=1 /\left[\sigma^{2}\left(F_{0}^{2}\right)+(0.020 P)^{2}+0.2753 P\right]$

where $P=\left(F_{\mathrm{o}}{ }^{2}+2 F_{\mathrm{c}}{ }^{2}\right) / 3$

$(\Delta / \sigma)_{\max }=0.001$

$\Delta \rho_{\max }=0.72 \mathrm{e} \AA^{-3}$

$\Delta \rho_{\min }=-0.80$ e $\AA^{-3}$

Extinction correction: SHELXL, $\mathrm{Fc}^{*}=\mathrm{kFc}\left[1+0.001 \mathrm{xFc}^{2} \lambda^{3} / \sin (2 \theta)\right]^{-1 / 4}$

Extinction coefficient: 0.0021 (5)

\section{Special details}

Geometry. All e.s.d.'s (except the e.s.d. in the dihedral angle between two 1.s. planes) are estimated using the full covariance matrix. The cell e.s.d.'s are taken into account individually in the estimation of e.s.d.'s in distances, angles and torsion angles; correlations between e.s.d.'s in cell parameters are only used when they are defined by crystal symmetry. An approximate (isotropic) treatment of cell e.s.d.'s is used for estimating e.s.d.'s involving 1.s. planes.

Refinement. Refinement of $F^{2}$ against ALL reflections. The weighted $R$-factor $w R$ and goodness of fit $S$ are based on $F^{2}$, conventional $R$-factors $R$ are based on $F$, with $F$ set to zero for negative $F^{2}$. The threshold expression of $F^{2}>\sigma\left(F^{2}\right)$ is used only for calculating $R$-factors(gt) etc. and is not relevant to the choice of reflections for refinement. $R$-factors based on $F^{2}$ are statistically about twice as large as those based on $F$, and $R$ - factors based on ALL data will be even larger.

Fractional atomic coordinates and isotropic or equivalent isotropic displacement parameters $\left(\AA^{2}\right)$

\begin{tabular}{llllll}
\hline & $x$ & $y$ & $z$ & $U_{\text {iso }} * / U_{\text {eq }}$ & Occ. $(<1)$ \\
\hline $\mathrm{Ba}$ & $0.547869(12)$ & 0.7500 & $0.74171(2)$ & $0.00734(5)$ & \\
$\mathrm{Mg}$ & $0.29439(5)$ & $-0.11139(4)$ & $0.20677(10)$ & $0.00705(9)$ & $0.9850(1)$ \\
$\mathrm{Mn}$ & $0.29439(5)$ & $-0.11139(4)$ & $0.20677(10)$ & $0.00705(9)$ & $0.0150(1)$ \\
$\mathrm{A} 1$ & $0.09176(4)$ & $0.40084(3)$ & $0.12947(8)$ & $0.00401(8)$ & \\
$\mathrm{P} 1$ & $0.15736(6)$ & 0.7500 & $0.68481(10)$ & $0.00467(9)$ & \\
P2 & $0.33413(4)$ & $0.44282(3)$ & $0.70566(7)$ & $0.00495(7)$ & \\
O1 & $0.27909(16)$ & 0.7500 & $0.9471(3)$ & $0.0072(2)$ & \\
O2 & $0.23251(16)$ & 0.7500 & $0.4303(3)$ & $0.0068(2)$ & \\
O3 & $0.05983(11)$ & $0.64525(9)$ & $0.6850(2)$ & $0.00791(18)$ & \\
O4 & $0.36649(12)$ & $0.55738(9)$ & $0.6050(2)$ & $0.00846(18)$ & \\
O5 & $0.25965(11)$ & $0.45434(9)$ & $0.9697(2)$ & $0.00811(18)$ & \\
O6 & $0.22678(12)$ & $0.38012(9)$ & $0.4741(2)$ & $0.00917(18)$ & \\
O7 & $0.47653(12)$ & $0.37189(9)$ & $0.7901(2)$ & $0.00821(18)$ & \\
OH8 & $0.12478(17)$ & 0.2500 & $0.0077(3)$ & $0.0083(3)$ & \\
OH9 & $0.06100(12)$ & $0.55814(9)$ & $0.1891(2)$ & $0.00679(17)$ & \\
H1 & $0.137(4)$ & 0.2500 & $-0.147(8)$ & $0.030^{*}$ & \\
H2 & $0.046(3)$ & $0.585(2)$ & $0.325(5)$ & $0.030^{*}$ & \\
& & & &
\end{tabular}

Atomic displacement parameters $\left(\AA^{2}\right)$

\begin{tabular}{lllllll}
\hline & $U^{11}$ & $U^{22}$ & $U^{33}$ & $U^{12}$ & $U^{13}$ & $U^{23}$ \\
\hline $\mathrm{Ba}$ & $0.00667(6)$ & $0.00826(7)$ & $0.00724(6)$ & 0.000 & $0.00171(4)$ & 0.000 \\
$\mathrm{Mg}$ & $0.0069(2)$ & $0.0070(2)$ & $0.00715(19)$ & $0.00042(16)$ & $0.00119(16)$ & $-0.00064(16)$
\end{tabular}


supporting information

$\begin{array}{lllllll}\text { Mn } & 0.0069(2) & 0.0070(2) & 0.00715(19) & 0.00042(16) & 0.00119(16) & -0.00064(16) \\ \text { A1 } & 0.00373(17) & 0.00395(17) & 0.00440(16) & -0.00027(13) & 0.00091(13) & 0.00002(13) \\ \text { P1 } & 0.00489(19) & 0.0050(2) & 0.00428(18) & 0.000 & 0.00138(15) & 0.000 \\ \text { P2 } & 0.00499(14) & 0.00536(15) & 0.00459(14) & 0.00024(11) & 0.00113(11) & 0.00027(10) \\ \text { O1 } & 0.0072(6) & 0.0085(6) & 0.0055(6) & 0.000 & 0.0001(5) & 0.000 \\ \text { O2 } & 0.0088(6) & 0.0061(6) & 0.0062(6) & 0.000 & 0.0036(5) & 0.000 \\ \text { O3 } & 0.0082(4) & 0.0069(4) & 0.0095(4) & -0.0029(3) & 0.0040(3) & -0.0015(3) \\ \text { O4 } & 0.0101(4) & 0.0070(4) & 0.0083(4) & -0.0001(3) & 0.0018(3) & 0.0023(3) \\ \text { O5 } & 0.0084(4) & 0.0102(5) & 0.0067(4) & -0.0008(4) & 0.0037(3) & -0.0006(3) \\ \text { O6 } & 0.0093(4) & 0.0100(5) & 0.0073(4) & -0.0002(4) & -0.0008(3) & -0.0015(3) \\ \text { O7 } & 0.0058(4) & 0.0087(5) & 0.0102(4) & 0.0021(3) & 0.0018(3) & 0.0020(3) \\ \text { OH8 } & 0.0110(6) & 0.0071(6) & 0.0071(6) & 0.000 & 0.0027(5) & 0.000 \\ \text { OH9 } & 0.0078(4) & 0.0076(4) & 0.0049(4) & -0.0001(3) & 0.0012(3) & -0.0016(3) \\ & & & & & \end{array}$

Geometric parameters $\left(\AA,{ }^{\circ}\right)$

\begin{tabular}{|c|c|c|c|}
\hline $\mathrm{Ba}-\mathrm{O}^{\mathrm{i}}$ & $2.7669(10)$ & $\mathrm{Mg}-\mathrm{O} 5^{\mathrm{x}}$ & $2.2090(12)$ \\
\hline $\mathrm{Ba}-\mathrm{O} 7^{\mathrm{ii}}$ & $2.7669(10)$ & $\mathrm{Al}-\mathrm{O} 3^{\mathrm{xi}}$ & $1.8523(11)$ \\
\hline $\mathrm{Ba}-\mathrm{O} 1$ & $2.7744(14)$ & $\mathrm{Al}-\mathrm{O} 6$ & $1.9080(11)$ \\
\hline $\mathrm{Ba}-\mathrm{O} 4$ & $2.8370(11)$ & $\mathrm{Al}-\mathrm{O} 5^{\mathrm{xii}}$ & $1.9287(10)$ \\
\hline $\mathrm{Ba}-\mathrm{O} 4^{\mathrm{iii}}$ & $2.8370(11)$ & $\mathrm{Al}-\mathrm{OH} 9$ & $1.9397(11)$ \\
\hline $\mathrm{Ba}-\mathrm{O}^{\mathrm{iv}}$ & $2.9019(11)$ & $\mathrm{Al}-\mathrm{OH} 9^{\mathrm{xiii}}$ & $1.9440(11)$ \\
\hline $\mathrm{Ba}-\mathrm{O}^{\mathrm{v}}$ & $2.9019(10)$ & $\mathrm{Al}-\mathrm{OH} 8$ & $1.9477(7)$ \\
\hline $\mathrm{Ba}-\mathrm{O} 2$ & $2.9566(15)$ & $\mathrm{P} 1-\mathrm{O} 2$ & $1.5232(14)$ \\
\hline $\mathrm{Ba}-\mathrm{OH} 8^{v}$ & $2.9658(15)$ & $\mathrm{P} 1-\mathrm{O} 1$ & $1.5278(15)$ \\
\hline $\mathrm{Ba}-\mathrm{O} 7^{\mathrm{v}}$ & $2.9661(10)$ & $\mathrm{P} 1-\mathrm{O} 3$ & $1.5321(10)$ \\
\hline $\mathrm{Ba}-\mathrm{O}^{\mathrm{iv}}$ & $2.9661(10)$ & $\mathrm{P} 1-\mathrm{O} 3^{\mathrm{iii}}$ & $1.5321(10)$ \\
\hline $\mathrm{Mg}-\mathrm{O} 4^{\mathrm{vi}}$ & $2.0490(11)$ & $\mathrm{P} 2-\mathrm{O} 4$ & $1.5083(11)$ \\
\hline $\mathrm{Mg}-\mathrm{O} 7^{\mathrm{vii}}$ & $2.0591(11)$ & $\mathrm{P} 2-\mathrm{O} 7$ & $1.5272(11)$ \\
\hline $\mathrm{Mg}-\mathrm{O} 1^{\text {viii }}$ & $2.0864(10)$ & $\mathrm{P} 2-\mathrm{O} 6$ & $1.5443(11)$ \\
\hline $\mathrm{Mg}-\mathrm{O} 2^{\mathrm{ix}}$ & $2.1227(10)$ & $\mathrm{P} 2-\mathrm{O} 5$ & $1.5680(10)$ \\
\hline $\mathrm{Mg}-\mathrm{OH} 9^{\mathrm{vi}}$ & $2.1729(11)$ & & \\
\hline $\mathrm{O} 4^{\mathrm{vi}}-\mathrm{Mg}-\mathrm{O} 7^{\mathrm{vii}}$ & $83.31(4)$ & $\mathrm{O} 3^{\mathrm{xi}}-\mathrm{Al}-\mathrm{OH} 9^{\mathrm{xiii}}$ & $89.97(5)$ \\
\hline $\mathrm{O} 4^{\mathrm{vi}}-\mathrm{Mg}-\mathrm{O} 1^{\mathrm{viii}}$ & $144.07(5)$ & $\mathrm{O} 6-\mathrm{Al}-\mathrm{OH} 9^{\mathrm{xiii}}$ & $170.24(5)$ \\
\hline $\mathrm{O}^{\text {vii }}-\mathrm{Mg}-\mathrm{O}^{\text {viii }}$ & $83.17(5)$ & $\mathrm{O}^{\mathrm{xii}}-\mathrm{Al}-\mathrm{OH} 9^{\mathrm{xiii}}$ & $94.31(5)$ \\
\hline $\mathrm{O} 4^{\mathrm{vi}}-\mathrm{Mg}-\mathrm{O} 2^{\mathrm{ix}}$ & $79.75(5)$ & $\mathrm{OH} 9-\mathrm{Al}-\mathrm{OH} 9^{\text {xiii }}$ & $77.04(5)$ \\
\hline $\mathrm{O} 7^{\mathrm{vii}}-\mathrm{Mg}-\mathrm{O} 2^{\mathrm{ix}}$ & $105.87(5)$ & $\mathrm{O}^{\mathrm{xi}}-\mathrm{Al}-\mathrm{OH} 8$ & $92.21(5)$ \\
\hline $\mathrm{O} 1^{\text {viii }}-\mathrm{Mg}-\mathrm{O} 2^{\mathrm{ix}}$ & $72.26(5)$ & $\mathrm{O} 6-\mathrm{Al}-\mathrm{OH} 8$ & $92.49(6)$ \\
\hline $\mathrm{O} 4^{\mathrm{vi}}-\mathrm{Mg}-\mathrm{OH} 9^{\mathrm{vi}}$ & $94.50(4)$ & $\mathrm{O}^{\mathrm{xii}}-\mathrm{Al}-\mathrm{OH} 8$ & $90.69(5)$ \\
\hline $\mathrm{O} 7^{\mathrm{vi}}-\mathrm{Mg}-\mathrm{OH} 9^{\mathrm{vi}}$ & $168.32(5)$ & $\mathrm{OH} 9-\mathrm{Al}-\mathrm{OH} 8$ & $170.59(5)$ \\
\hline $\mathrm{O} 1^{\text {viii }-\mathrm{Mg}-\mathrm{OH} 9^{\mathrm{vi}}}$ & $104.78(5)$ & $\mathrm{OH} 9^{\text {xiii }}-\mathrm{Al}-\mathrm{OH} 8$ & $96.50(6)$ \\
\hline $\mathrm{O} 2^{\text {ix }}-\mathrm{Mg}-\mathrm{OH} 9^{\text {vi }}$ & $84.93(5)$ & $\mathrm{O} 2-\mathrm{P} 1-\mathrm{O} 1$ & $109.67(8)$ \\
\hline $\mathrm{O} 4^{\mathrm{vi}}-\mathrm{Mg}-\mathrm{O} 5^{\mathrm{x}}$ & $102.80(5)$ & $\mathrm{O} 2-\mathrm{P} 1-\mathrm{O} 3$ & $109.74(5)$ \\
\hline $\mathrm{O} 7^{\mathrm{vii}}-\mathrm{Mg}-\mathrm{O} 5^{\mathrm{x}}$ & $97.57(4)$ & $\mathrm{O} 1-\mathrm{P} 1-\mathrm{O} 3$ & $108.60(5)$ \\
\hline $\mathrm{O} 1^{\text {viii }-\mathrm{Mg}-O 5^{\mathrm{x}}}$ & $111.89(4)$ & $\mathrm{O} 2-\mathrm{P} 1-\mathrm{O} 3^{\mathrm{iii}}$ & $109.74(5)$ \\
\hline $\mathrm{O} 2^{\mathrm{ix}}-\mathrm{Mg}-\mathrm{O} 5^{\mathrm{x}}$ & $156.55(5)$ & $\mathrm{O} 1-\mathrm{P} 1-\mathrm{O} 3^{\mathrm{iii}}$ & $108.60(5)$ \\
\hline $\mathrm{OH} 9^{\mathrm{vi}}-\mathrm{Mg}-\mathrm{O} 5^{\mathrm{x}}$ & $71.65(4)$ & $\mathrm{O} 3-\mathrm{P} 1-\mathrm{O} 3^{\mathrm{iii}}$ & $110.46(8)$ \\
\hline
\end{tabular}




$\begin{array}{llll}\mathrm{O}^{x i}-\mathrm{Al}-\mathrm{O} 6 & 85.88(5) & \mathrm{O} 4-\mathrm{P} 2-\mathrm{O} 7 & 113.41(6) \\ \mathrm{O} 3^{\mathrm{xi}}-\mathrm{Al}-\mathrm{O} 5^{\mathrm{xii}} & 174.52(5) & \mathrm{O} 4-\mathrm{P} 2-\mathrm{O} 6 & 109.59(6) \\ \mathrm{O} 6-\mathrm{Al}-\mathrm{O} 5^{\mathrm{xii}} & 89.36(5) & \mathrm{O} 7-\mathrm{P} 2-\mathrm{O} 6 & 107.74(6) \\ \mathrm{O} 3^{\mathrm{xi}}-\mathrm{Al}-\mathrm{OH} 9 & 94.60(5) & \mathrm{O} 4-\mathrm{P} 2-\mathrm{O} 5 & 109.04(6) \\ \mathrm{O} 6-\mathrm{Al}-\mathrm{OH} 9 & 94.47(5) & \mathrm{O} 7-\mathrm{P} 2-\mathrm{O} 5 & 106.57(6) \\ \mathrm{O} 5^{\mathrm{xii}}-\mathrm{Al}-\mathrm{OH} 9 & 83.07(5) & \mathrm{O} 6-\mathrm{P} 2-\mathrm{O} 5 & 110.45(6)\end{array}$

Symmetry codes: (i) $-x+1, y+1 / 2,-z+2$; (ii) $-x+1,-y+1,-z+2$; (iii) $x,-y+3 / 2, z$; (iv) $-x+1, y+1 / 2,-z+1$; (v) $-x+1,-y+1,-z+1$; (vi) $x,-y+1 / 2, z$; (vii) $-x+1, y-1 / 2,-z+1$; (viii) $x, y-1, z-1$; (ix) $x, y-1, z$; (x) $x,-y+1 / 2, z-1$; (xi) $-x,-y+1,-z+1$; (xii) $x, y, z-1$; (xiii) $-x,-y+1,-z$.

Hydrogen-bond geometry $\left(A,{ }^{\circ}\right)$

\begin{tabular}{lllll}
\hline$D-\mathrm{H} \cdots A$ & $D-\mathrm{H}$ & $\mathrm{H} \cdots A$ & $D \cdots A$ & $D-\mathrm{H} \cdots A$ \\
\hline $\mathrm{O} H 8-\mathrm{H} 1 \cdots \mathrm{O} 6^{\text {xii }}$ & $0.79(4)$ & $2.66(3)$ & $3.3180(16)$ & $142(1)$ \\
$\mathrm{O} H 9-\mathrm{H} 2 \cdots \mathrm{O} 3$ & $0.78(3)$ & $1.89(3)$ & $2.6512(13)$ & $166(3)$ \\
\hline
\end{tabular}

Symmetry code: (xii) $x, y, z-1$. 